\title{
Probiotics in 14-day triple therapy for Asian pediatric patients with Helicobacter pylori infection: a network meta-analysis
}

\author{
Juanjuan Wen ${ }^{1, *}$, Pailan Peng ${ }^{1,2, *}$, Pengfei Chen ${ }^{1,3, *}$, Lirong Zeng ${ }^{1}$, Qinghua Pan ${ }^{1}$, \\ Wenbin Wei ${ }^{1}$ and Jianhua $\mathrm{He}^{1}$ \\ ${ }^{1}$ Department of Gastroenterology, The Central Hospital of Enshi Autonomous Prefecture, Enshi, China \\ ${ }^{2}$ Department of Gastroenterology, Renmin Hospital of Wuhan University, Wuhan, China \\ ${ }^{3}$ Department of Gastroenterology, Zhongnan Hospital of Wuhan University, Wuhan, China \\ *These authors contributed equally to this work \\ Correspondence to: Wenbin Wei, email: weiwenbinenshi@sohu.com \\ Jianhua He, email: cpfwhu@163.com
}

Keywords: Helicobacter pylori, probiotics, pediatrics, 14-day triple therapy, network meta-analysis

Received: July 04, 2017

Accepted: September 21, 2017

Published: October 07, 2017

Copyright: Wen et al. This is an open-access article distributed under the terms of the Creative Commons Attribution License 3.0 (CC BY 3.0), which permits unrestricted use, distribution, and reproduction in any medium, provided the original author and source are credited.

\section{ABSTRACT}

Although 14-day triple therapy has been widely administrated for eradicating Helicobacter pylori (H. pylori) in Asia, its antibiotic-associated side effects restrict the effectivity of eradication therapy in pediatric patients. Therefore, a network meta-analysis (NMA) was conducted to compare the efficacy and safety of probiotics supplemented in 14-day triple therapy in Asian pediatric patients.

Materials and Methods: Randomized controlled trials (RCTs) were retrieved comprehensively in electronic databases (such as PubMed, Cochrane library, Embase, CNKI, Wan fang database, VIP database and CBM) until April 2017. Additional references were obtained from reviewed articles. NMA was performed using a randomeffects model under a frequentist framework.

Results: Seventeen RCTs were included. NMA indicated that Bifidobacterium infantis+Clostridium butyricum was most beneficial for $\boldsymbol{H}$. pylori eradication rates ( $\mathrm{P}$-score $=\mathbf{0 . 8 2}$ ) and Bacillus mesentericus+Clostridium butyricum+Streptococcus faecalis for total side effects (P-score $=0.77$ ). Taken together, Bacillus mesentericus+Clostridium butyricum+Streptococcus faecalis was the best one to supplement in 14-day triple therapy due to its efficacy $(P$-score $=0.72$ ) and safety (P-score $=0.77)$. Additionally, pairwise meta-analysis indicated that probiotics supplemented 14-day triple therapy significantly increased $\boldsymbol{H}$. pylori eradication rates (RR: 1.16, 95\%CI: 1.07-1.26) and reduced the incidence of total side effects (RR: $0.40,95 \% \mathrm{CI}: 0.34-0.48$ ) compared with placebo.

Conclusions: Bacillus mesentericus+Clostridium butyricum+Streptococcus faecalis is the optimal probiotic regime of reducing total side effects and improving eradication rates when supplemented 14-day triple therapy. Further direct evidence is needed to warrant it.

\section{INTRODUCTION}

In contrast with developed countries, Helicobacter pylori (H. pylori) infections occur earlier in life and with a higher prevalence in the developing world [1,2]. In parts of the developing world, especially in Asian countries, the prevalence of $H$. pylori infection increased with age and exceeded $80 \%$ by 19 years of age [2]. For instance, in Bangladesh, the prevalence of $H$. pylori was already $58 \%$ by 4 years of age and quickly rose to $82 \%$ by 9 years of age [2]. Similar findings were reported from pediatric studies in other Asian countries including India, Sri Lanka and China $[1,3,4]$. Children infected with $H$. pylori could develop many complications and symptoms like recurrent 
abdominal pain, dyspepsia, iron deficiency anemia, chronic gastritis, peptic ulcers and gastric cancer [5]. H. pylori infection has also been reported to cause a wide variety of extra-digestive manifestations, such as sudden infant death syndrome and short stature [6]. H. pylori eradication not only can cure peptic ulcers temporarily, but also reduce the lifetime risk of gastric cancer in long term due to increasing longevity in developing countries [7].

Because $H$. pylori is a bacterium, antibiotics certainly as a therapeutic agent will be administrated to eradicate it, like recent triple therapy. Nevertheless, recently accepted triple therapies still demonstrate failure rates as high as $20 \%$ to $30 \%$ due to increasing clarithromycin and metronidazole resistances as well as poor tolerability and reduced compliance [7-9]. A longer treatment duration may increase eradication rates that suggests an increase to 14 days instead of 7 days [7]. There is a strong recommendation by Joint ESPGHAN/ NASPGHAN Guidelines for 14-day triple therapy as firstline therapy for pediatric patients with $H$. pylori infection where clarithromycin and metronidazole resistances are presenting commonly in developing countries [10]. In addition, supplementary probiotics can reduce side effects and improve efficacy of $H$. pylori eradication therapies [11-16].

Although these studies suggest that there may be a benefit for reduction of side effects and improved efficacy when added to $H$. pylori eradication therapies, the evidence from Asia is limited and the same meaningful conclusions amongst these different probiotic strains seemed not be obtained as well as for 14-day triple therapy. Therefore, network meta-analysis (NMA) was performed to compare the efficacy and safety of specific probiotic supplementations in 14-day triple therapy for Asian pediatric population.

\section{MATERIALS AND METHODS}

We performed and reported the results of this pairwise and NMA in accordance with Preferred Reporting Items for Systematic Reviews and Meta-Analyses (PRISMA) statements [17, 18].

\section{Literature research}

We conducted a comprehensive search on databases including PubMed, Cochrane library, Embase and 4 Chinese Database [China National Knowledge Infrastructure (CNKI), database of Wan fang, VIP database and the Chinese Biomedical Database(CBM)] until April 2017. Literature were identified with keywords including (Helicobacter pylori OR H. pylori OR HP), probiotics, (pediatrics OR children), (randomized controlled trial OR RCT OR clinical trial) and (triple therapy OR eradication therapy) (search strategy seen in Supplementary Files). Additionally, manual search for the references cited in reviews and meta-analyses was performed as well. There were no language restrictions.

\section{Inclusion and exclusion criteria}

We included the RCTs meeting following eligibility criteria: (i) clinical trials from Asia; (ii) pediatric patients with $H$. pylori infection; (iii) clinical trials comprising of 14-day triple therapy (proton pump inhibitor and two antibiotics) plus placebo or no extra intervention and the same triple therapy plus probiotics; (iv) $H$. pylori eradication was confirmed at least 4 weeks after therapy termination. Articles were excluded if (i) reviews or comments; (ii) articles without data of $H$. pylori eradication rates and/or side effects.

\section{Study selection}

The articles were screened and selected by two researchers (Pengfei Chen and Lirong Zeng) independently. Initially, records of duplicates were removed using Endnote 7.4 software (Thomson Reuters). Remaining abstracts and full texts were reviewing based on inclusion and exclusion criteria. Disagreements were resolved by discussions.

\section{Data extraction and quality assessment}

Two reviewers (Juanjuan Wen and Pailan Peng) independently extracted the relevant information from each eligible study depending on a pre-prepared data abstraction sheet. Data were including location and study design of trials, clinical characteristics and number of patients, initial and rechecking methods for conformation of $H$. pylori infection, strains and period of probiotic regimens, eradication regimens and outcome data. The quality of included studies was assessed using Jadad scale, including three items such as randomized ( 2 points), double-blinding ( 2 points) and withdrawals and drop outs (1 point) [19]. A Jadad score of 3 or higher was considered as high quality. Disagreements were resolved by discussions.

The primary outcomes were eradication rates of $H$. pylori and incidence of total side effects, and secondary outcomes included incidence of specific side effects. The specific side effects of interest were the common symptoms that appeared during treatment, including diarrhea, nausea or vomiting, taste disturbance, loss of appetite, bloating, headache, constipation, and abdominal pain.

\section{Data synthesis and analysis}

Conventional pairwise meta-analysis was initially carried out for the comparison between probiotic group and placebo group. The estimated effects using relative ratios (RRs) with 95\% confidence intervals (CIs) based on 
the random effects model were analyzed by STATA 11.0 software (Stata Corporation, College Station, TX, USA). Secondary, NMA based on the random effects model was performed by netmeta $\mathrm{R}$ package with a frequentist framework [20, 21]. The P-scores that were based solely on the point estimates and standard errors of the network estimates were calculated to rank all probiotic regimens and the higher P-score value was correspond to better result for respective probiotic regimen [22].

Publication bias was evaluated using the Egger's and Begg's tests $[23,24]$. If a $P$ value $<0.05$, a trim and fill method was further conducted and recalculated the pooled estimate iteratively, until the funnel plot was symmetrical around the (new) pooled estimate [25]. Additionally, heterogeneity was measured with Q statistic and $\mathrm{I}^{2}$ statistic $[26,27]$. If an $\mathrm{I}^{2}$ value of $>50 \%$ or a $P$ value $<0.05$, sensitivity analysis was conducted by excluding one individual study each time to assess the influence of each individual study on the pooled RRs.

\section{RESULTS}

\section{Study characteristics}

A systematic search yielded a total of 213 articles. After literature reviewing, 89 duplicates were removed and 50 reviews were excluded, respectively. Additionally, 22 articles were excluded due to not met included criteria. Finally, a total of 17 trials were identified (Figure 1) and listed in Supplementary Table 5 [28-44].

The included trials involving 1932 pediatric patients has investigated 10 probiotic regimens, such as (1) Bifidobacterium animalis (B. animalis)+Lactobacillus acidophilus(L. acidophilus) + L. casei; (2) Bacillus mesentericus (B. mesentericus)+Clostridium butyricum (C. butyricum)+Streptococcus faecalis ( $S$. faecalis); (3) B. bifidum + B. infantis $+L$. acidophilus $+L$. bulgaricus + L. casei + L. reuteri+ Streptococcus; (4) B. infantis + C. butyricum; (5) B. longum + Enterococcus faecalis (E. faecalis) + L. acidophilus; (6) B. longum $+L$. bulgaricus $+S$. thermophilus; (7) L. acidophilus; (8) L. acidophilus + B. bifidum; (9) L. delbrueckii+L. acidophilus +Lactococcuslactis (L. lactis); (10) Saccharomyces boulardii (S. boulardii). There were 15 trials from China, 1 from Turkey and 1 from Iran. Assessing by Jadad scale, the overall Jadad scores of study quality ranged from 2 to 4 and the median of Jadad scores was 3 .

\section{Conventional pairwise meta-analysis}

Compared with placebo, probiotics supplemented 14-day triple therapy significantly improved eradication rates of $H$. pylori (RR: 1.16, 95\% CI: 1.07-1.26, $\mathrm{I}^{2}=62.7 \%$ ) (Figure 2). After sensitivity analysis was performed by omitting the study by He AZ [32], adjusted RR was 1.19 (95\% CI: $\left.1.13-1.25, \mathrm{I}^{2}=0 \%\right)$. In term of safety, probiotics supplemented 14-day triple therapy could reduce incidence of total side effects compared with placebo (RR: 0.40, 95\% CI: 0.34-0.48, $\mathrm{I}^{2}=0 \%$ ) (Figure 3). Additionally, subgroup analyses for specific probiotics found that L. acidophilus, S. boulardii and several multi- strain probiotics could significantly enhance the effectiveness of 14-day triple therapy (more detail seen in Supplementary Tables 1-2).

\section{Network meta-analysis}

There were 9 and 8 probiotic regimens supplemented 14-day triple therapy reporting data of eradication rates and total side effects, respectively (Figure 4). In term of improving eradication rates of $H$. pylori, B. infantis $+C$. butyricum was the best to add in 14-day triple therapy $(\mathrm{P}$-score $=0.82)$. B. mesentericus $+C$. butyricum $+S$. faecalis was most beneficial for total side effects (P-score $=0.77$ ). Taken together, B. mesentericus $+C$. butyricum $+S$. faecalis was the best one to supplement in 14-day triple therapy due to its efficacy $(\mathrm{P}$-score $=0.72)$ and safety $(\mathrm{P}$-score $=0.77)$ (Figure 5$)$. The relative effectiveness of all possible pairs of probiotics was presented in league tables (Supplementary Tables 3-4).

As for subtypes of side effects, B. bifidum $+B$. infantis $+L$. acidophilus $+L . \quad$ bulgaricus $+L . \quad$ casei $+L$. reuteri+ Streptococcus was most beneficial for reducing the incidence of diarrhea (P-score $=0.80), L$. acidophilus $+B$. bifidum for nausea/vomiting (P-score $=0.79), B$. mesentericus $+C$. butyricum $+S$. faecalis for loss of appetite (P-score $=0.73)$, L. acidophilus for constipation (P-score $=0.71)$, L acidophilus for taste disturbance $(\mathrm{P}$-score $=0.76)$, L. acidophilus + B. bifidumfor Abdominal pain $(\mathrm{P}-$ score $=0.89), S$. boulardii for bloating $(\mathrm{P}$-score $=0.76)$, L. acidophilus for headache $(\mathrm{P}$-score $=$ 0.78) (Figure 6).

\section{Publication bias}

Publication bias was assessed using data of $H$. pylori eradication rates. Visual inspection of the Begg's funnel plot found a slightly asymmetrical distribution (Figure 7A), which indicated having potential publication bias $(P=0.08)$. By trimming and inputting 5 studies using the trim and fill method (Figure 7B), the recalculated pooled RR for H. pylori eradication rates was 1.20 (95\% CI: 1.12-1.29) (Supplementary Figures 1-2), which was not significantly changed from the initial estimate (RR: 1.16, 95\% CI: 1.07-1.26).

\section{DISCUSSION}

This complexed meta-analysis included RCTs all from Asian countries, where appears high prevalence rate of $H$. pylori infection [7]. Our meta-analysis showed that probiotic supplementation can statistically benefit 
14-day triple therapy with both eradication rates and safety. Amongst included probiotics, B. infantis $+C$. butyricum was best for eradication rates of $H$. pylori and $B$. mesentericus $+C$. butyricum $+S$. faecalis best for reducing the incidence of total side effects. Additionally, it further found that beneficial effects of probiotics are strain specific. For example, as an addition in 14-day triple therapy, B. bifidum $+B$. infantis $+L$. acidophilus $+L$. bulgaricus + L. casei + L. reuteri + Streptococcus could reduce the incidence of diarrhea, which is the most common side effect associated with antibiotics. Given the results from this study, probiotics as a supplementation to 14-day triple therapy are considered as an alternative treatment regimen. Moreover, specific probiotics are administrated for corresponding specific side effect.
Probiotics, namely probiotic bacteria, have defined as live microorganisms, which most distribute in anterointernal mucosa and skin, confer a health benefit for the host. In infectious disease, probiotics act in the gastrointestinal luminal environment by reducing luminal $\mathrm{pH}$, inhibiting adhesion of other harmful microorganisms and antagonizing pathogenic bacteria by secreting bacteriocins [45]. Kabir AM and colleagues [46] found that $L$. salivarius was against and inhibited colonization of $H$. pylori, when supplemented in eradication therapy may be enhance the eradication of H. pylori. L. salivarius inhibited both the attachment and IL-8 release of $H$. pylori in vitro. $H$. pylori could not colonize the stomach of L. salivarius infected gnotobiotic BALB/c mice, but colonized in large numbers and subsequently caused active

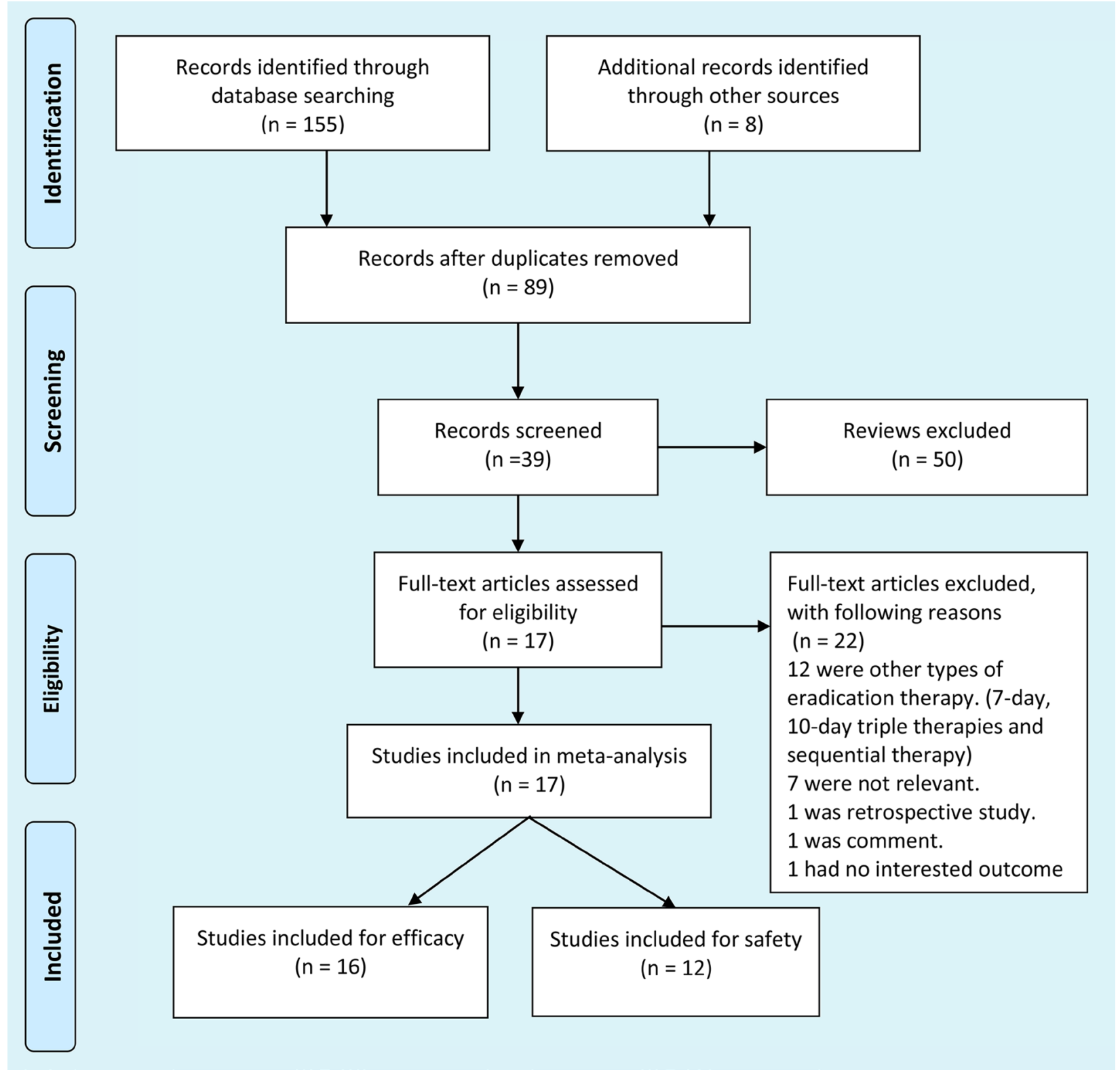

Figure 1: Identification process for eligible trials. 
gastritis in germ free mice. In addition, L. salivarius given after $H$. pylori implantation could eliminate colonization of H. pylori [46]. The conventional meta-analysis by Li S et al. in children demonstrate that probiotics supplementation has efficacy for H. pylori eradication therapy [14], which was in line with our conventional meta-analysis; but without more information on specific probiotics. Lately, two network meta-analyses have revealed the comparative effectiveness on probiotics supplementation for $H$. pylori eradication therapy $[47,48]$. One network meta-analysis suggested that L. acidopilus was a slightly better choice for triple therapy of 7 days and 14 days in adult patients, and $S$. boulardii was more applicable for 10-day triple therapy [48]. Another network meta-analysis of pediatric population suggested that, to supplemented triple therapy, L. casei was the best for $H$. pylori eradication rates, and multi-strain probiotics ( $L$. acidophilus plus $L$. rhamnosus) for total side effects [47]. These results of above network meta-analyses were different from our study, which might be greatly associated with different

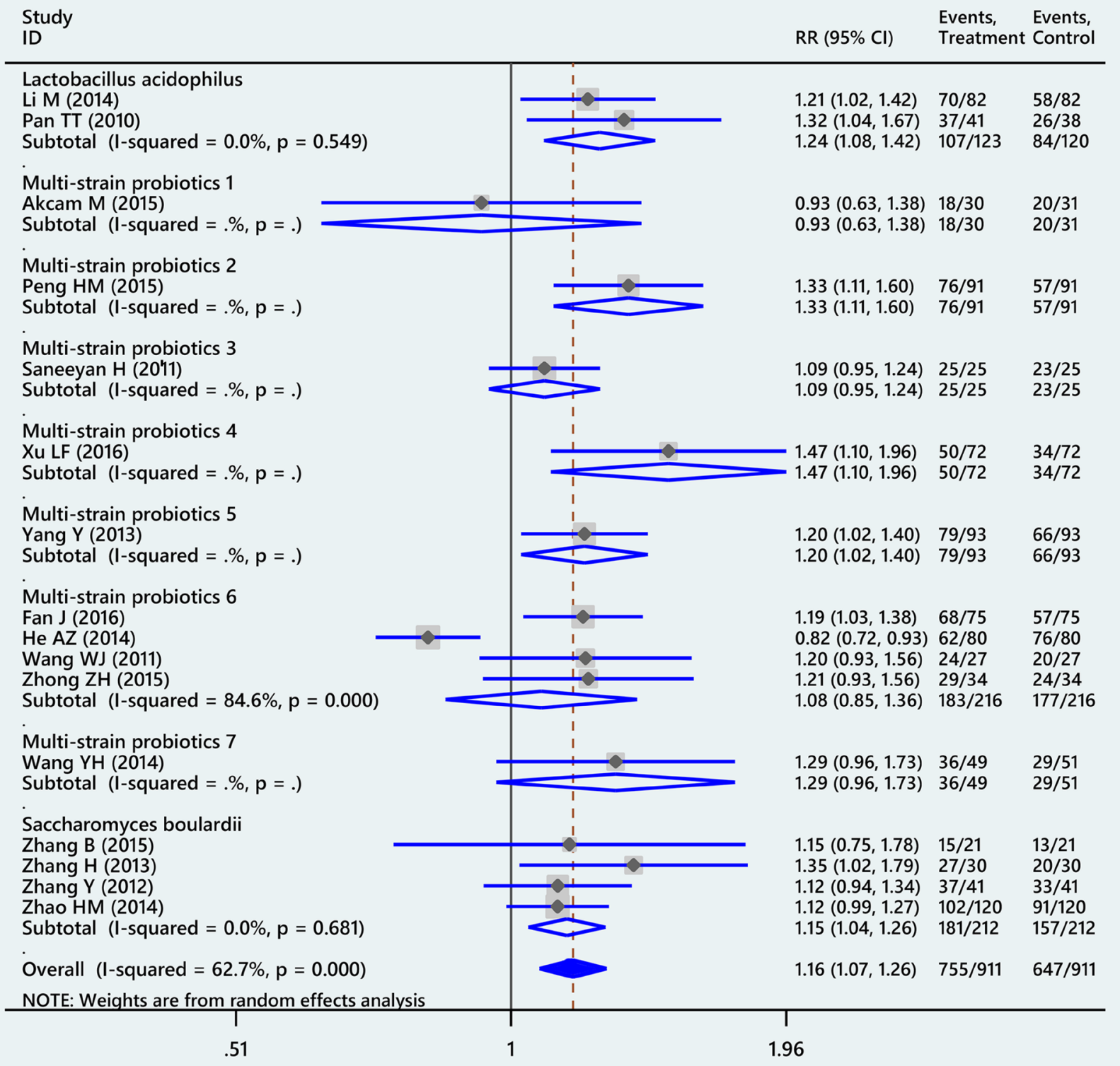

Figure 2: Forest plot of conventional meta-analysis for Helicobacter pylori eradication of probiotic regimens supplemented 14-day triple therapy compared with placebo. Multi-strain probiotics 1: Bifidobacterium animalis + Lactobacillus acidophilus +Lactobacillus casei; Multi-strain probiotics 2: Bacillus mesentericus +Clostridium butyricum +Streptococcus faecalis; Multistrain probiotics 3: Bifidobacterium bifidum + Bifidobacterium infantis + Lactobacillus acidophilus + Lactobasillus bulgaricus + Lactobasillus casei, Lactobasillus reuteri+Streptococcus; Multi-strain probiotics 4: Bifidobacterium infantis+Clostridium butyricum; Multi-strain probiotics 5: Bifidobacterium longum+Enterococcus faecalis +Lactobacillus acidophilus; Multi-strain probiotics 6: Bifidobacterium longum +Lactobacillus bulgaricus + Streptococcus thermophilus; Multi-strain probiotics 7: Lactobacillus acidophilus + Bifidobacterium bifidum; Multi-strain probiotics 8: Lactobacillus delbrueckii+Lactobacillus acidophilus +Lactococcus lactis). 
population and different area. Additionally, these two network meta-analysis also included some studies from non-Asian counties, which generally used the probiotics of Lactobacillus species. However, less studies of Lactobacillus species were included in our network metaanalysis, which maybe weaken the strength of evidence on Lactobacillus species when ranking the effectiveness among different probiotics.

In 2017, the Joint ESPGHAN/NASPGHAN Guidelines has recommended 14-day triple therapy as first-line therapy for pediatric patients with $H$. pylori infection because of clarithromycin and metronidazole resistances in developing countries [10]. In our study, probiotics, especially $B$. infantis $+C$. butyricum, as addition could be a new choice for enhancing 14-day triple therapy to eradicate $H$. pylori in children.

Apart from the efficacy of eradication therapy, antibiotic-associated side effects and bacterial resistance cannot be neglected because these factors could lead to the poor tolerability of drugs and hinder patients' compliance, and consequently cause anti-H. pylori treatment failure in clinical practice [49]. In our study,

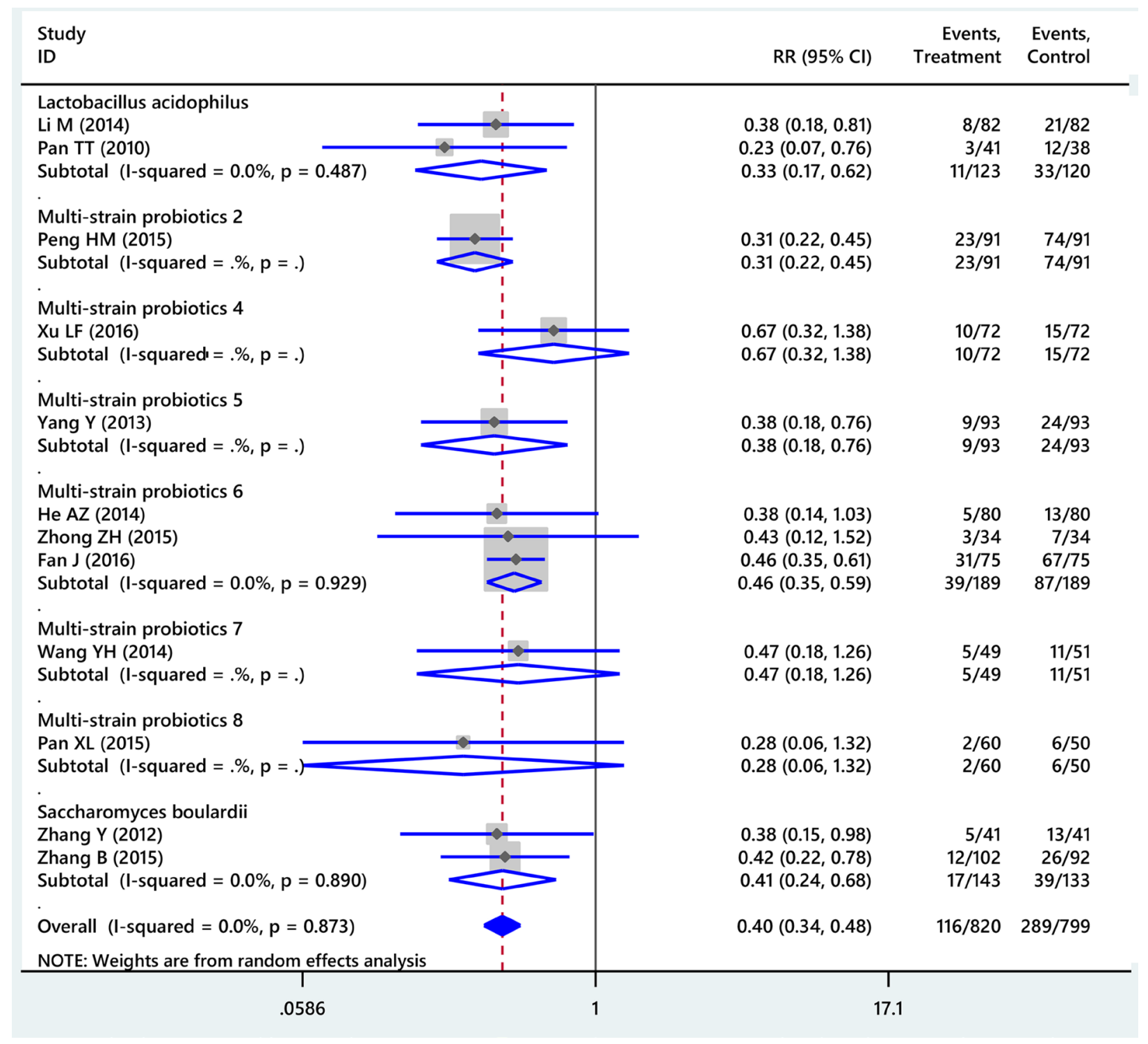

Figure 3: Forest plot of conventional meta-analysis for total side effects of probiotic regimens supplemented 14-day triple therapy compared with placebo. Multi-strain probiotics 1: Bifidobacterium animalis + Lactobacillus acidophilus + Lactobacillus casei; Multi-strain probiotics 2: Bacillus mesentericus+Clostridium butyricum+Streptococcus faecalis; Multi-strain probiotics 3: Bifidobacterium bifidum+Bifidobacterium infantis + Lactobacillus acidophilus + Lactobasillus bulgaricus + Lactobasillus casei, Lactobasillus reuteri+Streptococcus; Multi-strain probiotics 4: Bifidobacterium infantis +Clostridium butyricum; Multi-strain probiotics 5: Bifidobacterium longum +Enterococcus faecalis +Lactobacillus acidophilus; Multi-strain probiotics 6: Bifidobacterium longum+Lactobacillus bulgaricus + Streptococcus thermophilus; Multi-strain probiotics 7: Lactobacillus acidophilus + Bifidobacterium bifidum; Multi-strain probiotics 8: Lactobacillus delbrueckii+Lactobacillus acidophilus+Lactococcus lactis). 


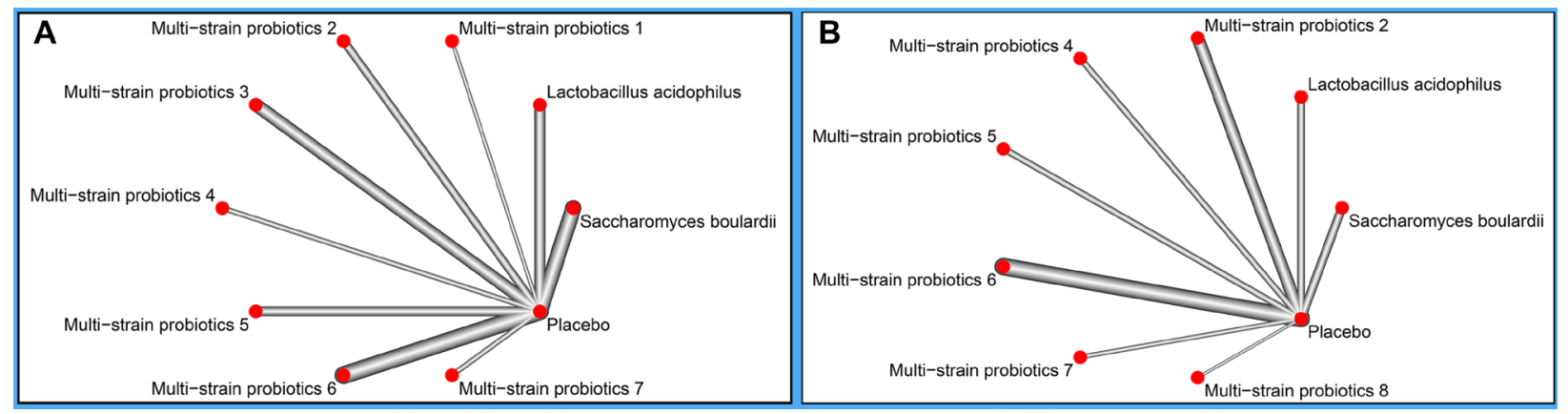

Figure 4: Network plots of probiotic regimens included in network meta-analysis. (A) Network relations of probiotic regimens that supplemented 14-day triple therapy on Helicobacter pylori eradication. (B) Network relations of probiotic regimens that supplemented 14-day triple therapy on total side effects. The nodes correspond to and are labeled according to the probiotic regimens, and the edges show which probiotic regimens are directly compared. The thickness of the lines is proportional to the inverse standard error of the direct comparisons. Multi-strain probiotics 1: Bifidobacterium animalis + Lactobacillus acidophilus + Lactobacillus casei; Multi-strain probiotics 2: Bacillus mesentericus + Clostridium butyricum + Streptococcus faecalis; Multi-strain probiotics 3: Bifidobacterium bifidum + Bifidobacterium infantis+Lactobacillus acidophilus+Lactobasillus bulgaricus+Lactobasillus casei, Lactobasillus reuteri+Streptococcus; Multistrain probiotics 4: Bifidobacterium infantis +Clostridium butyricum; Multi-strain probiotics 5: Bifidobacterium longum +Enterococcus faecalis+Lactobacillus acidophilus; Multi-strain probiotics 6: Bifidobacterium longum+Lactobacillus bulgaricus + Streptococcus thermophilus; Multi-strain probiotics 7: Lactobacillus acidophilus+Bifidobacterium bifidum; Multi-strain probiotics 8: Lactobacillus delbrueckii+Lactobacillus acidophilus + Lactococcus lactis).

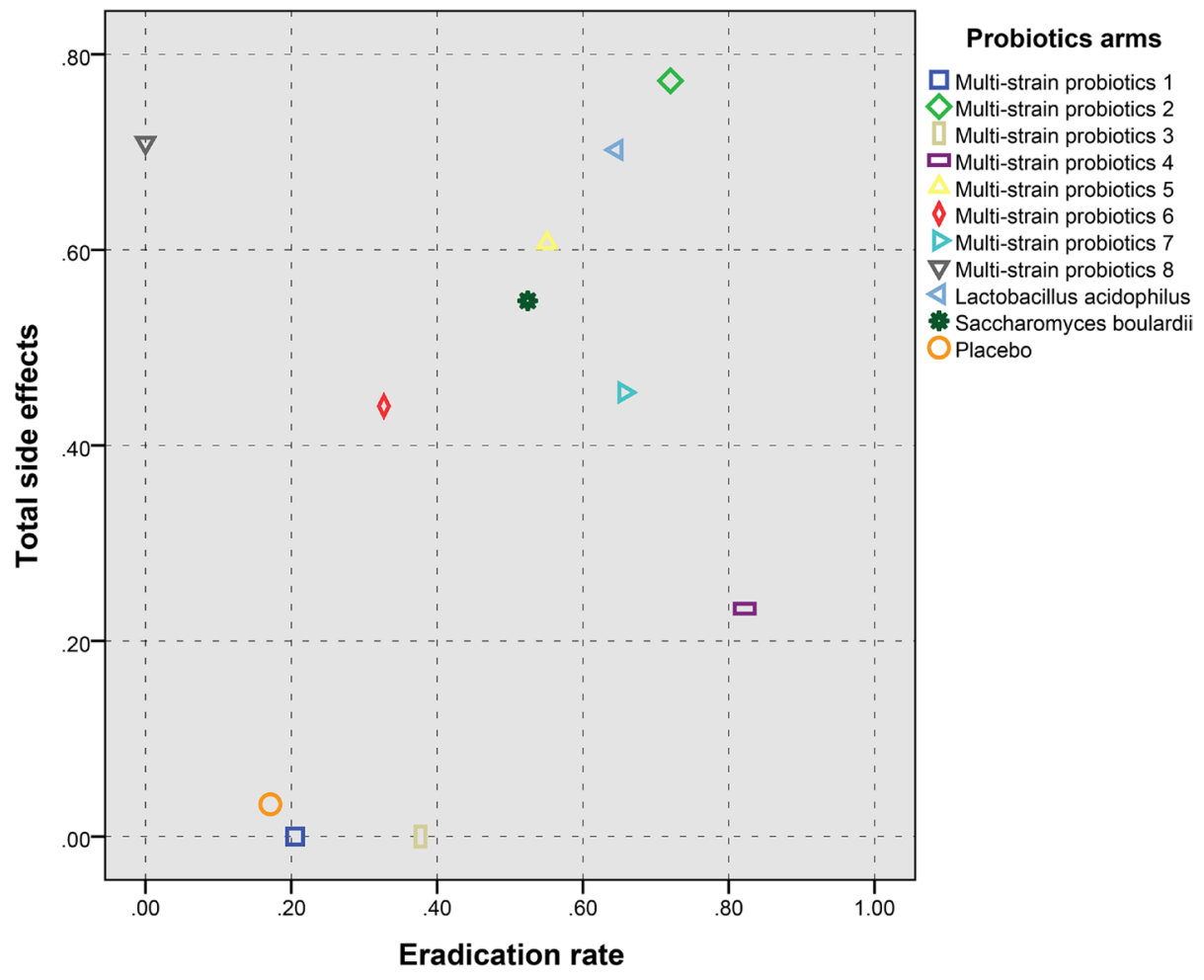

Figure 5: The scatter plot based on P-scores for total side effects (Y-axis) and Helicobacter pylori eradication (X-axis) of probiotic regimens supplemented 14-day triple therapy. The P-scores are larger and better, and if $\mathrm{P}$-score $=0$, this parameter is missing. Therefore, Multi-strain probiotics 2 is the better result for the probiotic regimen supplemented 14-day triple therapy with both total side effects and Helicobacter pylori eradication rate. Multi-strain probiotics 1: Bifidobacterium animalis + Lactobacillus acidophilus +Lactobacillus casei; Multi-strain probiotics 2: Bacillus mesentericus + Clostridium butyricum + Streptococcus faecalis; Multistrain probiotics 3: Bifidobacterium bifidum +Bifidobacterium infantis + Lactobacillus acidophilus + Lactobasillus bulgaricus + Lactobasillus casei, Lactobasillus reuteri+Streptococcus; Multi-strain probiotics 4: Bifidobacterium infantis +Clostridium butyricum; Multi-strain probiotics 5: Bifidobacterium longum+Enterococcus faecalis +Lactobacillus acidophilus; Multi-strain probiotics 6: Bifidobacterium longum+Lactobacillus bulgaricus + Streptococcus thermophilus; Multi-strain probiotics 7: Lactobacillus acidophilus + Bifidobacterium bifidum; Multi-strain probiotics 8: Lactobacillus delbrueckii+Lactobacillus acidophilus+Lactococcus lactis). 


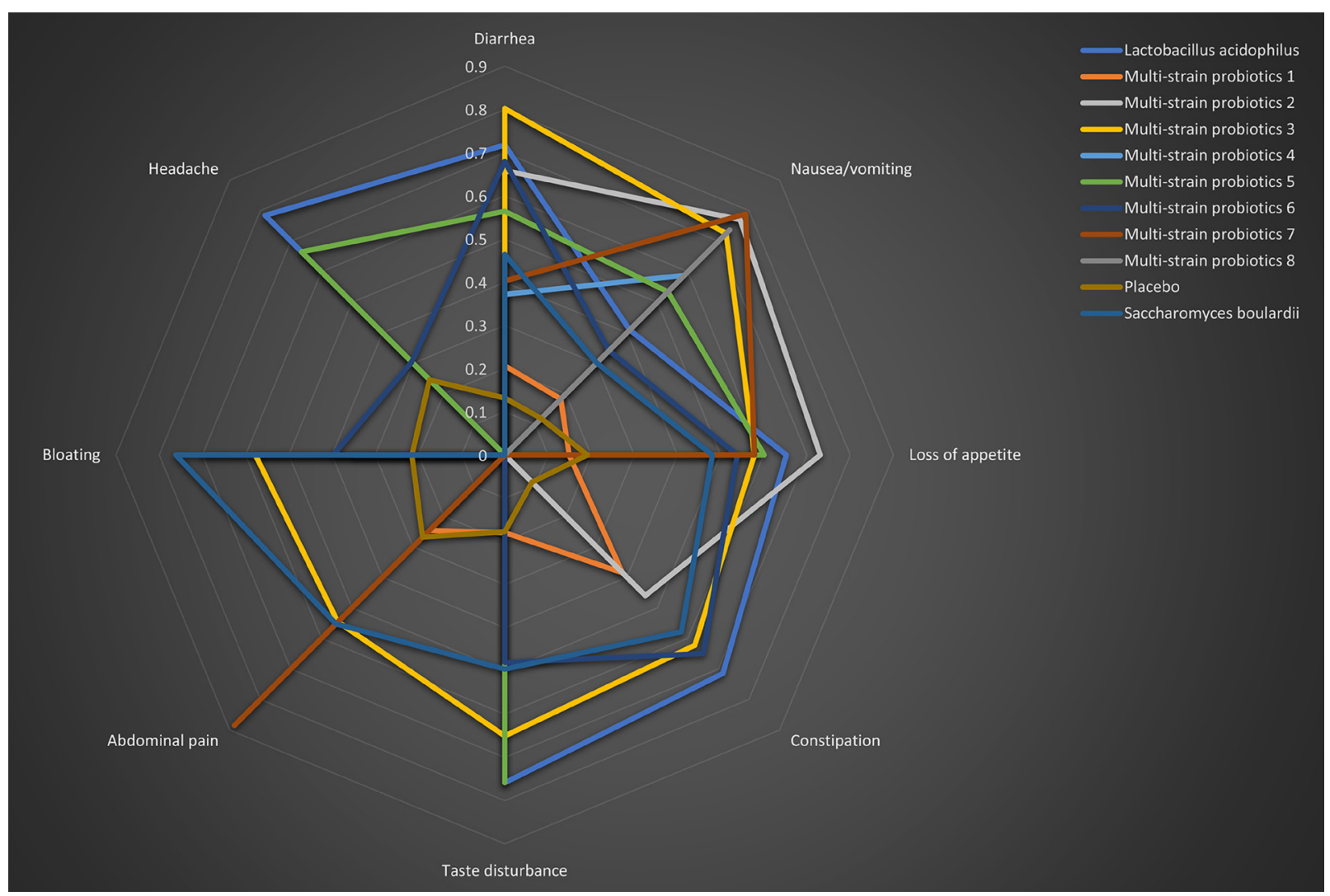

Figure 6: Radar chart based on P-scores for subtypes of side effects of probiotic regimens supplemented 14-day triple therapy. Different probiotic regimens are shown in different colors. The axes of this radar chart are oriented in the way that the best values for a particular parameter are positioned outside. Therefore, the better results for a probiotic regimen the bigger area covered by the polygon for this probiotic regimen on this radar chart. In addition, the P-scores are larger and better, and if P-score $=0$, this parameter is missing. Multi-strain probiotics 1: Bifidobacterium animalis + Lactobacillus acidophilus + Lactobacillus casei; Multi-strain probiotics 2: Bacillus mesentericus + Clostridium butyricum + Streptococcus faecalis; Multi-strain probiotics 3: Bifidobacterium bifidum + Bifidobacterium infantis+Lactobacillus acidophilus+Lactobasillus bulgaricus+Lactobasillus casei, Lactobasillus reuteri+Streptococcus; Multi-strain probiotics 4: Bifidobacterium infantis + Clostridium butyricum; Multi-strain probiotics 5: Bifidobacterium longum +Enterococcus faecalis+Lactobacillus acidophilus; Multi-strain probiotics 6: Bifidobacterium longum+Lactobacillus bulgaricus +Streptococcus thermophilus; Multi-strain probiotics 7: Lactobacillus acidophilus+Bifidobacterium bifidum; Multi-strain probiotics 8: Lactobacillus delbrueckii+Lactobacillus acidophilus+Lactococcus lactis).

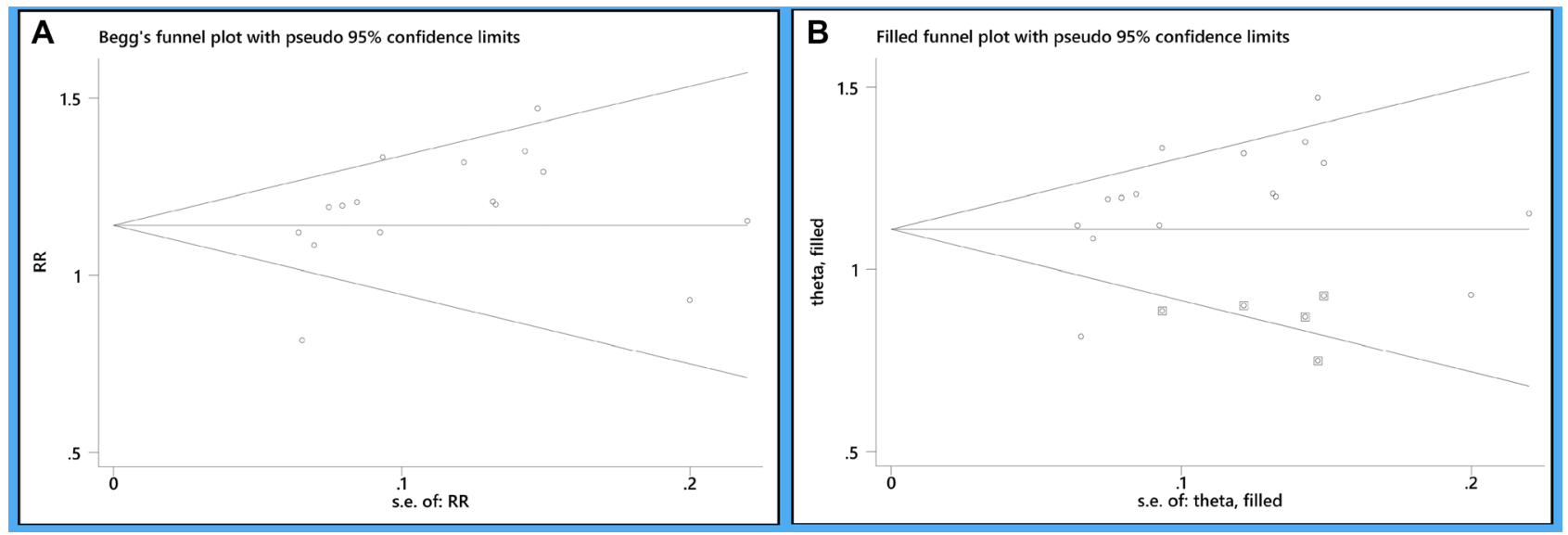

Figure 7: (A) Asymmetrical funnel plot of $H$. pylori eradication rates; (B) trim and fill method for previous asymmetrical funnel plot of $H$. pylori eradication rates. The squares represent the adjusted studies. 
the occurrence of antibiotic-associated total side effects was significantly reduced by the addition of probiotics as well as specific probiotic stains like multi-strain probiotics (such as B. longum, L. bulgaricus and S. thermophilus), S. boulardii and L. acidophilus compared with the placebo (Supplementary Table 2).

This study has several strengths and weaknesses. We concentrated on clinical trials that all investigated14-day triple therapy in Asia where $H$. pylori infection have great impact on children healthy. So, such clinical conclusions for mongoloid race, a main population in Asia, can be firstly obtained. However, the methodologies of all RCTs were not rigorously carried out, which might affect the conclusions. Additionally, strengths of this complex metaanalysis were that the different strains or regimens of probiotics were separated for assessing efficacy and safety. Therefore, probiotics of most effectivity can be guided to add in 14-day triple therapy for the specific purpose, especially to prevent certain side effect. Nevertheless, most trials did not take antibiotic-associated side effects as key outcomes, and not documented more information (such as antibiotic resistant of $H$. pylori, initial treatment or second-line strategy for experiencing a failure of eradicating $H$. pylori, etc.).

All in all, our meta-analyses found that probiotics are beneficial for 14-day triple therapy in Asian pediatric patients with $H$. pylori infection. For obtaining greater benefit of improving eradication rates and reducing antibiotic-associated side effects, the mixture of B. mesentericus $+C$. butyricum $+S$. faecalis may be recommended to add in 14-day triple therapy. Further direct evidence is needed to warrant it.

\section{CONFLICTS OF INTEREST} interests.

The authors declare that they have no competing

\section{REFERENCES}

1. Graham DY, Adam E, Reddy GT, Agarwal JP, Agarwal R, Evans DJ Jr, Malaty HM, Evans DG. Seroepidemiology of Helicobacter pylori infection in India. Comparison of developing and developed countries. Dig Dis Sci. 1991; 36:1084-1088.

2. Frenck RW Jr, Clemens J. Helicobacter in the developing world. Microbes Infect. 2003; 5:705-713.

3. Mitchell HM, Li YY, Hu PJ, Liu Q, Chen M, Du GG, Wang ZJ, Lee A, Hazell SL. Epidemiology of Helicobacter pylori in southern China: identification of early childhood as the critical period for acquisition. J Infect Dis. 1992; 166:149-153.

4. Fernando N, Holton J, Vaira D, DeSilva M, Fernando D. Prevalence of Helicobacter pylori in Sri Lanka as determined by PCR. J Clin Microbiol. 2002; 40:2675-2676.

5. Zheng PY, Jones NL. Recent advances in Helicobacter pylori infection in children: from the petri dish to the playground. Can J Gastroenterol. 2003; 17:448-454.

6. Sherman PM, Macarthur C. Current controversies associated with Helicobacter pylori infection in the pediatric population. Front Biosci. 2001; 6:E187-192.

7. Hunt RH, Xiao SD, Megraud F, Leon-Barua R, Bazzoli F, van der Merwe S, Vaz Coelho LG, Fock M, Fedail S, Cohen H, Malfertheiner P, Vakil N, Hamid S, et al. Helicobacter pylori in developing countries. World Gastroenterology Organisation Global Guideline. J Gastrointestin Liver Dis. 2011; 20:299-304.

8. Vandenplas Y, Blecker U. Helicobacter pylori infection in children. Acta paediatrica. 1998; 87:1105-1112.

9. Drouin E. Helicobacter pylori: Novel therapies. Can J Gastroenterol. 1999; 13:581-583.

10. Jones NL, Koletzko S, Goodman K, Bontems P, Cadranel S, Casswall T, Czinn S, Gold BD, Guarner J, Elitsur Y, Homan M, Kalach N, Kori M, et al. Joint ESPGHAN/ NASPGHAN Guidelines for the Management of Helicobacter pylori in Children and Adolescents (Update 2016). J Pediatr Gastroenterol Nutr. 2017; 64:991-1003.

11. Lu MH, Yu S, Deng JQ, Yan Q, Yang C, Xia GD, Zhou X. Efficacy of Probiotic Supplementation Therapy for Helicobacter pylori Eradication: A Meta-Analysis of Randomized Controlled Trials. PLoS One. 2016; 11:26.

12. McFarland LV, Huang $Y$, Wang L, Malfertheiner $P$. Systematic review and meta-analysis: Multi-strain probiotics as adjunct therapy for Helicobacter pylori eradication and prevention of adverse events. United European Gastroenterol J. 2016; 4:546-561.

13. McFarland LV, Malfertheiner P, Huang Y, Wang L. Metaanalysis of single strain probiotics for the eradication of Helicobacter pylori and prevention of adverse events. World J Metaanal. 2015; 3:97-117.

14. Li S, Huang XL, Sui JZ, Chen SY, Xie YT, Deng Y, Wang J, Xie L, Li TJ, He Y, Peng QL, Qin X, Zeng ZY. Meta-analysis of randomized controlled trials on the efficacy of probiotics in Helicobacter pylori eradication therapy in children. Eur J Pediatr. 2014; 173:153-161.

15. Zheng X, Lyu L, Mei Z. Lactobacillus-containing probiotic supplementation increases Helicobacter pylori eradication rate: evidence from a meta-analysis. Rev Esp Enferm Dig. $2013 ; 105: 445-453$.

16. Szajewska H, Horvath A, Piwowarczyk A. Meta-analysis: the effects of Saccharomyces boulardii supplementation on Helicobacter pylori eradication rates and side effects during treatment. Aliment Pharmacol Ther. 2010; 32:1069-1079.

17. Hutton B, Salanti G, Caldwell DM, Chaimani A, Schmid CH, Cameron C, Ioannidis JP, Straus S, Thorlund K, Jansen JP, Mulrow C, Catala-Lopez F, Gotzsche PC, et al. The PRISMA extension statement for reporting of systematic reviews incorporating network meta-analyses of health care interventions: checklist and explanations. Ann Intern Med. $2015 ; 162: 777-784$.

18. Liberati A, Altman DG, Tetzlaff J, Mulrow C, Gotzsche PC, Ioannidis JP, Clarke M, Devereaux PJ, Kleijnen J, Moher D. The PRISMA statement for reporting systematic 
reviews and meta-analyses of studies that evaluate health care interventions: explanation and elaboration. PLoS Med. 2009; 6:e1000100.

19. Jadad AR, Moore RA, Carroll D, Jenkinson C, Reynolds DJ, Gavaghan DJ, McQuay HJ. Assessing the quality of reports of randomized clinical trials: is blinding necessary? Control Clin Trials. 1996; 17:1-12.

20. Schwarzer G, Carpenter J, Rucker G. Meta-Analysis with R (Use-R!). Switzerland: Springer International Publishing. 2015.

21. Rucker G. Network meta-analysis, electrical networks and graph theory. Res Synth Methods. 2012; 3:312-324.

22. Rucker G, Schwarzer G. Ranking treatments in frequentist network meta-analysis works without resampling methods. BMC Med Res Methodol. 2015; 15:58.

23. Begg CB, Mazumdar M. Operating characteristics of a rank correlation test for publication bias. Biometrics. 1994; 50:1088-1101.

24. Egger M, Smith GD, Schneider M, Minder C. Bias in metaanalysis detected by a simple, graphical test. BMJ. 1997; 315:629-634.

25. Duval S, Tweedie R. Trim and Fill: A Simple Funnel-PlotBased Method of Testing and Adjusting for Publication Bias in Meta-Analysis. Biometrics. 2000; 56:455.

26. Deeks JJ. Issues in the selection of a summary statistic for meta-analysis of clinical trials with binary outcomes. Stat Med. 2002; 21:1575-1600.

27. Higgins JP, Thompson SG, Deeks JJ, Altman DG. Measuring inconsistency in meta-analyses. BMJ. 2003; 327:557-560.

28. Pan T, Zhu H, Lu H. Clinical observation on the effect of probiotics in the treatment of Helicobacter pylori infection in children. Strait Pharma J. 2010; 22:109-110.

29. Wang W, Luo H, Wei J. Effect of probiotics on Helicobacter pylori eradication in children with abdominal pain. Chin J Microecol. 2011; 23:916-917.

30. Zhang Y, Li J. Effect of Saccharomyces boulardii on treatment of Helicobacter pylori infection in children of triple therapy. J Clin Pediatr. 2012; 30:928-931.

31. Yang Y, Huang J, Shao S. Efficacy of probiotics combined with triple therapy in treatment of Helicobacter pylori infection in children. Chin J Nosocomiol. 2013; 23:5757-5758.

32. Zhang H. Clinical effect about Boulardii powder combined triple therapy treating Helicobacter pylori infection in children. Medical J Chin People Health. 2013; 25:38-40.

33. He A. Efficacy of probiotics combined with triple therapy in treatment of Helicobacter pylori infection in children. World Health Digest. 2014; 14:122.

34. Li M, Zhou X. Efficacy of triple therapy combined with probiotics in the treatment of Helicobacter pylori infection in children. Modern J Integr Trad Chin and West Med. 2014; 23:2883-2884.

35. Peng H, Mei H, Wang B. Efficacy of eradication therapy containing probiotics on Helicobacter pylori. J Chin Physician. 2015; 17:1249-1251.
36. Zhong H, Ye Y, Lv B. Clinical observation of the application of probiotics combined with triple therapy in the treatment of Helicobacter pylori infection in children. Chin Med Engineering. 2015; 23:144-146.

37. Fan J, Li M, Liao J. Probiotics combined with triple therapy in the treatment of Helicobacter pylori infection in children. J Modern Clin Med. 2016.

38. $\mathrm{Xu} \mathrm{L}$, Yang $\mathrm{X}$, Guo J. Randomized controlled trial of Clostridium butyricum in eradication treatment of Helicobacter pylori infection in children. Chin J Microecol. 2016; 28:413-416.

39. Pan X. Effect of probiotics combined with triple therapy clinical value in treatment of Helicobacter pylori associated chronic gastritis. J Qiqihar University Med. 2015; 36:3627-3629.

40. Saneeyan H, Layegh S, Rahimi H. Effectivness of probiotic on treatment of Helicobacter pylori infection in children. Journal of Isfahan Medical School. 2011; 29:882-889.

41. Wang YH, Huang Y. Effect of Lactobacillus acidophilus and Bifidobacterium bifidum supplementation to standard triple therapy on Helicobacter pylori eradication and dynamic changes in intestinal flora. World J Microbiol Biotechnol. 2014; 30:847-853.

42. Zhao HM, Ou-Yang HJ, Duan BP, Xu B, Chen ZY, Tang J, You JY. [Clinical effect of triple therapy combined with Saccharomyces boulardii in the treatment of Helicobacter pylori infection in children]. [Article in Chinese]. Zhongguo dang dai er ke za zhi. 2014; 16:230-233.

43. Akcam M, Koca T, Salman H, Karahan N. The effects of probiotics on treatment of Helicobacter pylori eradication in children. Saudi Med. 2015; 36:286-290.

44. Bin Z, Ya-Zheng X, Zhao-Hui D, Bo C, Li-Rong J, Vandenplas Y. The Efficacy of Saccharomyces boulardii CNCM I-745 in Addition to Standard Helicobacter pylori Eradication Treatment in Children. Pediatr Gastroenterol Hepatol Nutr. 2015; 18:17-22.

45. Dylag K, Hubalewska-Mazgaj M, Surmiak M, Szmyd J, Brzozowski T. Probiotics in the mechanism of protection against gut inflammation and therapy of gastrointestinal disorders. Curr Pharm Des. 2014; 20: 1149-1155.

46. Kabir AM, Aiba Y, Takagi A, Kamiya S, Miwa T, Koga Y. Prevention of Helicobacter pylori infection by lactobacilli in a gnotobiotic murine model. Gut. 1997; 41:49-55.

47. Feng JR, Wang F, Qiu X, McFarland LV, Chen PF, Zhou R, Liu J, Zhao Q, Li J. Efficacy and safety of probioticsupplemented triple therapy for eradication of Helicobacter pylori in children: a systematic review and network metaanalysis. Eur J Clin Pharmacol. 2017.

48. Wang F, Feng J, Chen P, Liu X, Ma M, Zhou R, Chang Y, Liu J, Li J, Zhao Q. Probiotics in Helicobacterpylori eradication therapy: Systematic review and network metaanalysis. Clin Res Hepatol Gastroenterol. 2017.

49. Deltenre M, Ntounda R, Jonas C, De Koster E. Eradication of Helicobacter pylori: why does it fail? Ital J Gastroenterol Hepatol. 1998; 30:S326-328. 